\title{
Dados sociodemográficos e condições de saúde de idosas inscritas no PSF de Maceió, AL
}

Sociodemographic data and health conditions in elderly enrolled in Family Health Program in Maceio city, Brazil

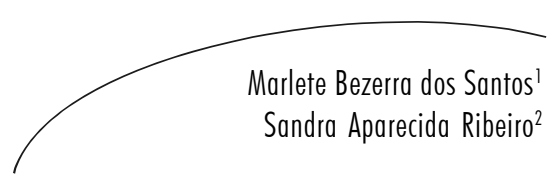

Resumo

Objetivos: Descrever as variáveis sociodemográficas e identificar as condições de saúde de mulheres idosas inscritas no Programa de Saúde da Família de Maceió. Métodos: Estudo descritivo, transversal, por amostragem, utilizando variáveis sociodemográficas e condições de saúde. Para avaliar o estado nutricional: índice de massa corporal (IMC) e circunferência da cintura (CC). A depressão foi avaliada por meio da GDS-30, a capacidade funcional por meio da escala de Lawton e o IPAQ, versão curta, para avaliar o nível de atividade física. Resultados: A amostra do estudo foi composta por 268 mulheres, com média de idade de 66,68 $\pm 5,77$ anos; destas, 70,5\% estavam na faixa entre 60-69 anos, 35,1\% moravam com o cônjuge e $48,5 \%$ viviam com menos de um salário mínimo per capita. Referiram de uma a duas morbidades $60,1 \%$ das idosas, sendo que a hipertensão arterial foi a mais frequente $(84,0 \%)$. A prevalência de obesidade foi de $21,6 \%$, enquanto $25,7 \%$ da amostra apresentaram baixo-peso e $67,9 \%$ tinham CC $\geq 80 \mathrm{~cm}$. Houve suspeita de depressão leve ou moderada em 41,0\%, 12,7\% apresentaram dependência parcial para as atividades instrumentais da vida diária e 50,7\% eram sedentárias ou insuficientemente ativas. Conclusões: Espera-se que esses resultados contribuam para melhor quantificar as necessidades dessa população, fornecendo informações para formulação de políticas públicas pelos gestores. Além disso, promover ações para garantir que aqueles que vivam mais de 60 anos possam fazê-lo com dignidade e qualidade de vida.

\footnotetext{
$1 \quad$ Faculdade de Nutrição. Universidade Federal de Alagoas

2 Departamento de Medicina Preventiva. Universidade Federal de São Paulo Apoio: Fundação de Amparo à Pesquisa do Estado de Alagoas (FAPEAL). Processo no 099/ 11386345.
}

Palavras-chave: Saúde do idoso. Envelhecimento. Perfil de saúde. Estado nutricional. Programa de Saúde da Família. 
Abstract

Objectives: To describe the sociodemographic variables and to identify the health conditions of elderly women enrolled in the Family Health Program in Maceio city, Brazil. Methods: The study is a descriptive, transversal and random sampling, using socio-demographic and health conditions variables. To evaluate the nutritional status were used: body mass index (BMI) and waist circumference (WC). Depression was evaluated using the GDS-30, functional capacity for means of the Lawton scale and to assess the physical activity level the IPAQ short version. Results: The sample of the study was composed of 268 women with the average age of $66.68 \pm 5.77$ years, being $70,5 \%$ from $60-69$ years old. In this sample $35.1 \%$ lived with their spouse and $48.5 \%$ lived on less than minimum salary; $60.1 \%$ of the elderly reported one to two morbidities, being hypertension the most frequent (84.0\%). Prevalence of obesity was $21.6 \%$ while $25.7 \%$ of the sample was underweight and $67.9 \%$ have WC $\geq 80 \mathrm{~cm} ; 41.0 \%$ were suspected of having mild or moderate depression, $12.7 \%$ showed partial dependence to instrumental activities of daily living and $50.7 \%$ were sedentary. Conclusions: We expect that these findings will contribute to a better quantification of needs of this population, providing information to public policies. In addition, it may promote actions to ensure that those aged 60 years or more can live with dignity and quality of life.
Key words: Elderly Health. Aging. Health profile. Nutritional status. Family Health Program.

\section{INTRODUÇÃO}

No Brasil, durante algum tempo, alimentouse o conceito de país jovem, enquanto, a partir de 1960, com o declínio da taxa de fecundidade, as projeções apontam para o crescimento da população idosa. ${ }^{1} \mathrm{~A}$ previsão é que, no período de 1950 a 2025, esta população cresça 16 vezes, contra cinco vezes a população total. Para 2050, as projeções são que, pela primeira vez na história, a população idosa com 60 anos ou mais irá superar o número de jovens, segundo dados do Instituto Brasileiro de Geografia e Estatística (IBGE). ${ }^{2}$

Em Maceió, capital de Alagoas, há uma população de 936.314 habitantes, dos quais $7,1 \%$ são formados por idosos, sendo $60,9 \%$ do sexo feminino (IBGE). ${ }^{3}$ A expectativa de vida no estado é de 67,2 anos, enquanto para o sexo feminino é de 71,3 anos. ${ }^{3}$ A taxa de cobertura de planos de assistência médica é de $23,2 \%$, de acordo com dados da Agência Nacional de Saúde Suplementar (ANS); em consequência, cerca de $76,8 \%$ utilizam exclusivamente os serviços do Sistema Único de Saúde (SUS). ${ }^{4}$ A cobertura do Programa de Saúde da Família (PSF), hoje Estratégia de Saúde da Família (ESF), no município de Maceió, é de $26,88 \%$ da população. ${ }^{3}$
O crescente aumento da categoria idosa em nível mundial tem provocado, nos órgãos governamentais e na sociedade, o desafio de mudanças médico-sociais próprias para o envelhecimento populacional. Para Veras et al. ${ }^{5}$, no Brasil os esforços ainda são pontuais e desarticulados. Ramos ${ }^{6}$ afirma que o sistema de saúde terá que fazer frente a uma crescente demanda por procedimentos diagnósticos e terapêuticos das doenças crônicas nãotransmissíveis, principalmente as cardiovasculares e as neurovegetativas, e a uma demanda ainda maior por serviços de reabilitação física e mental.

Pinheiro \& Travassos $^{7}$ consideram que os indivíduos nos extremos da cadeia etária usam mais os serviços de saúde que a população em geral. Além do mais, a população idosa tem mais necessidade de procedimentos de alto custo. As mulheres são as maiores consumidoras dos serviços de saúde.

As condições de saúde da população idosa podem ser determinadas por meio de seus perfis. Entre estes, destacam-se: percepção da saúde, morbidade referida, estado nutricional, depressão e independência para as atividades cotidianas. As grandes desigualdades regionais 
sociodemográficas, culturais e de saúde são pouco relatadas em estudos epidemiológicos de base populacional envolvendo o idoso, sobretudo no Nordeste e, por conseguinte, em Maceió. ${ }^{8}$

Em razão de os estudos apontarem que as mulheres frequentam mais o sistema de saúde que os homens, optou-se pela escolha do gênero feminino. ${ }^{8-10}$ Dentro desse contexto, o presente estudo tem o objetivo de descrever as variáveis sociodemográficas e as condições de saúde de mulheres idosas inscritas no Programa de Saúde da Família de Maceió.

\section{MÉTODOS}

Trata-se de um estudo do tipo descritivo, de delineamento transversal, por amostragem. Os procedimentos utilizados para a seleção da amostra levaram em consideração múltiplos estágios, com o intuito de se obter uma amostragem estratificada aleatória que fosse representativa da população feminina idosa inscrita nas unidades básicas de saúde (UBS) com o modelo de PSF de Maceió, totalizando 36 unidades e 72 equipes de saúde, segundo as listas fornecidas pela Secretaria Municipal de Saúde de Maceió.

Os parâmetros para o cálculo da amostra tiveram por base: o tamanho da população de 7.886 idosas inscritas no PSF/Maceió, nível de confiança de $95 \%$, erro alfa de $5 \%$, poder do teste estatístico de $80 \%$. Desse modo, a amostra mínima consistiu de 226 idosas. Para compensar possíveis perdas, a amostra foi acrescida em $20 \%$, atingindo 271 pesquisadas, sendo entrevistadas 268 . A perda foi de $1,1 \%$, que correspondeu a uma UBS com o PSF que se encontrava em reforma (houve vários contatos durante o período de coleta). Nessa unidade havia a previsão de três respondentes.

A estratificação aconteceu segundo o critério de probabilidade proporcional ao número de idosas inscritas no modelo. O número de prontuários de família a ser examinado no arquivo se deu por amostragem sistemática, calculada com base no "n" mínimo, acrescido da proporção de mulheres na faixa de 60 a 79 anos, obtendo-se um intervalo amostral igual a quatro. A partir daí, por meio da amostragem aleatória simples, a cada quatro prontuários de família um era retirado para compor a amostra em cada UBS/PSF. Não havendo idosa que atendesse aos critérios de pesquisa, automaticamente era retirado um novo prontuário. Durante o curso da pesquisa, houve 87 idosas $(32,4 \%)$ que não puderam ou não quiseram comparecer e foram substituídas.

O critério de inclusão foram mulheres com idades entre 60 e 79 anos cadastradas nas equipes de PSF de Maceió. Foram excluídas do estudo as portadoras de deficiências físicas e as que se recusaram a participar ou assinar o termo de consentimento livre e esclarecido (TCLE).

O trabalho de campo foi realizado entre julho de 2008 a fevereiro de 2009, por três pesquisadoras associadas, especialmente treinadas por um projeto piloto e pela pesquisadora principal. A pesquisa consistiu na aplicação de um questionário que continha as variáveis sociodemográficas (idade, grau de instrução, estado civil, arranjo domiciliar e renda per capita), variáveis relacionadas às condições de saúde (percepção da saúde, morbidade referida, tabagismo, estado nutricional, escala de depressão, capacidade funcional e nível de atividade física).

Para conhecer a percepção da saúde era indagado: "Como a senhora considera sua saúde: excelente, boa, regular ou ruim”. Em relação ao tabagismo, foi perguntado "a senhora fuma, sim ou não?". Para morbidade autorreferida, questionou-se sobre as seguintes doenças: hipertensão arterial, diabetes mellitus, doença do coração, artrite/reumatismo/artrose, problema gastrointestinal, doença crônica pulmonar, osteoporose, embolia/derrame e câncer.

Foram obtidas as variáveis antropométricas: índice de massa corporal (IMC), em $\mathrm{kg} / \mathrm{m}^{2}$, e circunferência da cintura (CC), em centímetros. ${ }^{11}$ Todas as medidas foram realizadas em duplicata. O IMC foi classificado segundo os valores propostos pela Organização Pan-Americana da 
Saúde (OPAS) no ano de 2002: baixo-peso (IMC $<23)$, eutrofia $(23 \leq \mathrm{IMC}<28)$, sobrepeso $(28 \leq \mathrm{IMC}<30)$ e obesidade $(\mathrm{IMC} \geq 30) .{ }^{12}$

A circunferência da cintura foi aferida seguindo-se os procedimentos descritos por Callaway et al. ${ }^{13}$ Para descrever a distribuição da obesidade, a medida CC foi agrupada de acordo com os pontos de corte para riscos de complicações metabólicas e cardiovasculares associadas à obesidade, adotado pela Organização Mundial de Saúde (2000) para as mulheres: adequado (CC $<80 \mathrm{~cm}$ ), elevado $(\mathrm{CC} \geq 80<88$ $\mathrm{cm})$ e muito elevado $(\mathrm{CC} \geq 88 \mathrm{~cm}) .{ }^{14}$

Para o rastreamento de sintomas depressivos, utilizou-se a Escala Geriátrica de Depressão (GDS), em sua versão composta por 30 itens, proposta por Yesavage et al..$^{15}$ e validada no Brasil por Stoppe Júnior et al. ${ }^{16}$ Os escores são: 0 a 10 é considerado normal; 11 a 20, depressão leve ou moderada e 21 a 30, depressão grave. Um escore acima de 10, com base nesses pontos de corte, indica a necessidade de confirmação de depressão.

O instrumento mais utilizado para avaliar a capacidade funcional de pessoas nãoinstitucionalizadas é a escala de Lawton e Brody. ${ }^{17}$ Por essa capacidade funcional, entenda-se aquela que se propõe a realizar sem dificuldade ou ajuda as atividades instrumentais da vida diária (AIVD), como preparo de refeições, tarefas domésticas, lavar roupa, manuseio de medicamentos, usar o telefone, manuseio de dinheiro, fazer compras e usar meios de transportes. Os escores variam de O a 24 pontos, de sorte que, quanto mais elevada a pontuação obtida pelo indivíduo, maior sua independência: de $\mathrm{O}$ a 8 pontos (dependência); $9 \mathrm{a}$ 16 pontos (dependência parcial) e 17 a 24 pontos (independência). ${ }^{17}$

O nível de atividade física foi determinado por meio do Questionário Internacional de Atividade Física (IPAQ), versão curta, validado na população brasileira por Matsudo et al. ${ }^{18} \mathrm{~A}$ forma curta foi recomendada para os estudos nacionais de prevalência e de possibilidade de comparação internacional. É um instrumento que permite estimar o tempo semanal gasto na realização de atividades físicas de intensidade moderada a vigorosa e em diferentes contextos da vida. Os dados foram coletados por meio de entrevista, tendo por referência a última semana. Para analisar os dados do IPAQ, consideraram-se quatro categorias: muito ativo ( $\geq 150 \mathrm{minutos} / \mathrm{semanal}$, todas as modalidades), ativo ( $\geq 150$ minutos/semanal, qualquer modalidade), irregularmente ativo $(\geq 10$ minutos/semanal) e sedentário ( $\leq 10$ minutos/ semanal), de acordo com os critérios propostos pelo consenso realizado entre o Centro de Estudos do Laboratório de Aptidão Física de São Caetano do Sul (CELAFISCS) e o Center for Disease of Prevention and Control (CDC) de Atlanta, em 2002. . $^{19-20}$

A análise estatística foi realizada com o programa SPSS para Windows (versão 13.0) e incluiu análise descritiva sob a forma de frequência, média, desvio-padrão e percentis (P25, P50 e P75).

O protocolo de pesquisa obteve aprovação do Comitê de Ética em Pesquisa da Universidade Federal de Alagoas/UFAL (n ${ }^{\circ}$ 015372/2006-85, aprovado em 26/01/2007) e da Universidade Federal de São Paulo/UNIFESP (nº 0332/08).

\section{RESULTADOS}

Foram estudadas 268 idosas, com média de idade de 66,68 \pm 5,77 anos. Houve maior proporção de idosas na faixa de 60 a 69 anos $(70,5 \%)$. Um total de $106(39,6 \%)$ eram viúvas e $35,1 \%$ eram casadas ou viviam com o companheiro As entrevistadas não alfabetizadas eram $41,8 \%$, enquanto que $22,8 \%$ tinham de 1 a 2 anos de estudo (tabela 1). 
Tabela 1 - Características sociodemográficas de idosas inscritas no Programa de Saúde da Família de Maceió. Maceió,AL, 2009.

\begin{tabular}{|c|c|c|}
\hline Variável & $(\mathrm{n}=268)$ & $\%$ \\
\hline \multicolumn{3}{|l|}{ Faixa etária } \\
\hline 60 a 69 & 189 & 70,5 \\
\hline 70 a 79 & 79 & 29,5 \\
\hline \multicolumn{3}{|l|}{ Estado conjugal } \\
\hline Solteira & 51 & 19,0 \\
\hline Cônjuge/Companheiro & 94 & 35,1 \\
\hline Viúva & 106 & 39,5 \\
\hline Desquitada & 1 & 0,4 \\
\hline Divorciada & 4 & 1,5 \\
\hline Separada & 12 & 4,5 \\
\hline \multicolumn{3}{|l|}{ Escolaridade } \\
\hline Não alfabetizada & 112 & 41,8 \\
\hline Alfabetizada & 156 & 58,2 \\
\hline \multicolumn{3}{|l|}{ Anos de estudo } \\
\hline Nenhum & 112 & 41,8 \\
\hline 1 a 2 & 61 & 22,8 \\
\hline 3 a 4 & 57 & 21,3 \\
\hline$\geq 5$ & 38 & 14,2 \\
\hline \multicolumn{3}{|l|}{ Arranjos domiciliares } \\
\hline Mora sozinha & 38 & 14,2 \\
\hline Cônjuge/Companheiro & 94 & 35,1 \\
\hline Filho(a) não casado (a) & 78 & 29,1 \\
\hline Filho(a) casado(a) & 39 & 14,6 \\
\hline Outros $^{1}$ & 19 & 7,0 \\
\hline \multicolumn{3}{|c|}{ Número de pessoas no domicílio } \\
\hline Nenhuma & 38 & 14,2 \\
\hline 1 a 2 & 110 & 41,0 \\
\hline 3 a 4 & 77 & 28,7 \\
\hline$\geq 5$ & 43 & 16,0 \\
\hline \multicolumn{3}{|l|}{ Renda per capita } \\
\hline$<1 \mathrm{SM}^{2}$ & 130 & 48,5 \\
\hline 1 a 2 SM & 94 & 35,1 \\
\hline$>2$ a $3 \mathrm{SM}$ & 26 & 9,7 \\
\hline$>3 \mathrm{SM}$ & 18 & 6,7 \\
\hline
\end{tabular}

1. Outros: irmãos, netos e sobrinhos.

2. $\mathbf{S M}=$ Salário Mínimo à época $=\mathrm{R} \$ 415,00$.

Ainda segundo a tabela 1, observou-se que em relação ao arranjo familiar no domicílio, $43,7 \%$ residiam com filhos não casados ou casados, $14,2 \%$ moravam sozinhase $69,7 \%$ residiam em casas com até quatro pessoas. Neste estudo, $48,5 \%$ das idosas viviam com renda percapita menor que um salário mínimo.
A tabela 2 trata da autopercepção da saúde, em que houve maior concentração de respostas "regular" (65,3\%). São fumantes $13,0 \%$, enquanto $50,4 \%$ nunca fumaram e $93,3 \%$ referem ter de uma a quatro morbidades. 
Tabela 2 - Distribuição de idosas inscritas no Programa de Saúde da Família de Maceió, segundo a autopercepção da saúde, tabagismo e número de comorbidades autorreferidas. Maceió,AL, 2009.

\begin{tabular}{lcc}
\hline \multicolumn{1}{c}{ Variável } & $(\mathrm{n}=268)$ & $\%$ \\
\hline Autopercepção da saúde & & \\
$\quad$ Excelente & 3 & 1,1 \\
Boa & 38 & 14,2 \\
Regular & 175 & 65,3 \\
Ruim & 52 & 19,4 \\
Tabagismo & & \\
Fumante & 35 & 13,0 \\
Ex-fumante & 98 & 36,8 \\
Nunca fumou & 135 & 50,4 \\
Número de comorbidades & & \\
Nenhuma & 6 & 2,2 \\
1 a 2 & 161 & 60,1 \\
3 a 4 & 89 & 33,2 \\
5 a 6 & 11 & 4,1 \\
$>6$ & 1 & 0,4 \\
\hline
\end{tabular}

Na tabela 3 está apresentada a distribuição das morbidades autorreferidas. A hipertensão arterial foi a mais prevalente $(84,0 \%)$, seguida por artrite/ reumatismo/artrose (56,7\%) e diabetes mellitus (31,3\%).

Tabela 3 - Frequências das morbidades autorreferidas em idosas inscritas no Programa de Saúde da Família de Maceió. Maceió,AL, 2009.

\begin{tabular}{lcccc}
\hline Morbidades autorreferidas & \multicolumn{2}{c}{$\operatorname{Sim}$} & \multicolumn{2}{c}{ Não } \\
\cline { 2 - 5 } & $(\mathrm{n})$ & $\%$ & $(\mathrm{n})$ & $\%$ \\
\hline Hipertensão arterial & 225 & 84,0 & 43 & 16,0 \\
Artrite/reumatismo/artrose & 152 & 56,7 & 116 & 43,3 \\
Diabetes mellitus & 84 & 31,3 & 184 & 68,7 \\
Doença do coração & 58 & 21,6 & 210 & 78,4 \\
Problema gastrointestinal & 49 & 18,3 & 219 & 81,7 \\
Osteoporose & 25 & 9,3 & 243 & 90,7 \\
Embolia/derrame & 10 & 3,7 & 258 & 96,3 \\
Doença crônica pulmonar & 5 & 1,9 & 263 & 98,1 \\
Câncer & 1 & 0,4 & 267 & 99,6 \\
\hline
\end{tabular}

A tabela 4 apresenta o estado nutricional de idosas matriculadas no PSF de Maceió. A frequência de eutrofia foi de $38,5 \%$, enquanto que $25,7 \%$ da amostra apresentaram baixo-peso. Obesidade $(21,6 \%)$ e sobrepeso $(14,2 \%)$ estavam presentes em $35,8 \%$ das pesquisadas. $\mathrm{O}$ estado de eutrofia foi mais frequente na faixa etária de 70-79 anos (41,8\%). A prevalência de obesidade entre as faixas etárias de 60-69 e 70-79 anos foi de $25,9 \%$ e $11,4 \%$, respectivamente. Acerca da obesidade abdominal, por meio da medida de circunferência da cintura, $67,9 \%$ da amostra 
apresentaram risco cardiovascular $(\mathrm{CC} \geq 80 \mathrm{~cm})$ elevado ou muito elevado. As idosas do grupo etário 70-79 anos foram classificadas com risco elevado $(22,8 \%)$ e $48,1 \%$ das idosas na faixa etária de 60-69 anos apresentaram risco muito elevado $(\mathrm{CC} \geq 88 \mathrm{~cm})$.

Tabela 4 - Frequência, média, desvio-padrão e percentil de idosas inscritas no Programa de Saúde da Família de Maceió, segundo o estado nutricional. Maceió,AL, 2009.

\begin{tabular}{|c|c|c|c|c|c|c|}
\hline \multirow[t]{2}{*}{ Variáveis antropométricas } & \multicolumn{2}{|c|}{ Variáveis } & \multirow[t]{2}{*}{ Média $\pm \mathrm{DP}^{1}$} & \multicolumn{3}{|c|}{ Percentil } \\
\hline & $\mathrm{n}$ & $\%$ & & 25 & 50 & 75 \\
\hline \multicolumn{7}{|l|}{ IMC (Kg/m²) } \\
\hline 60-69 anos & 189 & 70,5 & $26,74 \pm 5,05$ & 23,20 & 27,00 & 30,05 \\
\hline Baixo peso & 45 & 23,8 & & & & \\
\hline Eutrofia & 70 & 37,0 & & & & \\
\hline Sobrepeso & 25 & 13,3 & & & & \\
\hline Obesidade & 49 & 25,9 & & & & \\
\hline 70-79 anos & 79 & 29,5 & $25,50 \pm 4,40$ & 22,40 & 25,10 & 28,10 \\
\hline Baixo peso & 24 & 30,4 & & & & \\
\hline Eutrofia & 33 & 41,8 & & & & \\
\hline Sobrepeso & 13 & 16,4 & & & & \\
\hline Obesidade & 9 & 11,4 & & & & \\
\hline Total & 268 & 100,0 & $26,38 \pm 4,89$ & 22,80 & 26,40 & 29,20 \\
\hline Baixo peso & 69 & 25,7 & & & & \\
\hline Eutrofia & 103 & 38,5 & & & & \\
\hline Sobrepeso & 38 & 14,2 & & & & \\
\hline Obesidade & 58 & 21,6 & & & & \\
\hline \multicolumn{7}{|l|}{$\mathrm{CC}(\mathrm{cm})$} \\
\hline 60-69 anos & 189 & 70,5 & $86,74 \pm 11,91$ & 79,00 & 87,00 & 94,75 \\
\hline$<80$ & 54 & 28,6 & & & & \\
\hline$\geq 80<88$ & 44 & 23,3 & & & & \\
\hline$\geq 88$ & 91 & 48,1 & & & & \\
\hline 70-79 anos & 79 & 29,5 & $84,90 \pm 10,94$ & 77,00 & 84,00 & 92,00 \\
\hline$<80$ & 32 & 40,5 & & & & \\
\hline$\geq 80<88$ & 18 & 22,8 & & & & \\
\hline$\geq 88$ & 29 & 36,7 & & & & \\
\hline Total & 268 & 100,0 & $86,19 \pm 11,64$ & 78,00 & 86,00 & 94,00 \\
\hline$<80$ & 86 & 32,1 & & & & \\
\hline$\geq 80<88$ & 62 & 23,1 & & & & \\
\hline$\geq 88$ & 120 & 44,8 & & & & \\
\hline
\end{tabular}

$\mathrm{Na}$ tabela 5, encontram-se as frequências da GDS-30, de sorte que $41,0 \%$ das pesquisadas foram classificadas com sintomas de depressão leve ou moderada e 4,1\%, grave;
$87,3 \%$ eram independentes funcionalmente, $12,7 \%$ apresentaram dependência funcional e $49,2 \%$ eram ativas ou muito ativas, segundo o IPAQ. 
Tabela 5 - Resultados do rastreamento para depressão, capacidade funcional e nível de atividade física em idosas inscritas no Programa de Saúde da Família de Maceió. Maceió,AL, 2009.

\begin{tabular}{lcc}
\hline \multicolumn{1}{c}{ Variável } & $\mathrm{n} \mathrm{(268)}$ & $\%(100)$ \\
\hline Depressão & & \\
Sem depressão & 147 & 54,9 \\
Depressão leve ou moderada & 110 & 41,0 \\
Depressão grave & 11 & 4,1 \\
Capacidade funcional & & \\
Dependência & - & - \\
Dependência moderada & 34 & 12,7 \\
Independente & 234 & 87,3 \\
Nível de atividade física & & \\
Sedentária & 52 & 19,4 \\
Insuficientemente ativa & 84 & 31,3 \\
Ativa & 130 & 48,5 \\
Muito ativa & 2 & 0,7 \\
\hline
\end{tabular}

\section{DISCUSSÃO}

O estudo envolve idosas inscritas no PSF de Maceió, entre 60 e 79 anos. Os resultados obtidos deverão ser interpretados levando-se em consideração as características da população estudada, restrita às idosas inscritas no PSF desse município, nesta faixa etária.

A faixa etária entre 60 a 69 anos apresenta maior proporção, quando comparada com o grupo etário de 70-79 anos. Em Maceió, 59,2\% da população idosa encontram-se na primeira faixa etária, e desta, $58,6 \%$ são do gênero feminino (IBGE). ${ }^{3}$ Outros estudos comprovam maior frequência de idosos neste grupo etário, bem como a feminização da população idosa. ${ }^{21-22}$

As idosas octogenárias do município de Maceió representam $1,06 \%$ da população, sendo o segmento que vem aumentando de maneira mais acentuada. ${ }^{23}$ Não podem compor a amostra pelo fato de este trabalho fazer parte de uma pesquisa maior, em que há necessidade de valores normativos brasileiros para a categoria, inexistentes até então.

Este estudo tem como limitações o fato de estar restrito às idosas de até 79 anos, pela falta de padrão antropométrico de referência nacional para idosos e falta de instrumento para aferição de memória.

A proporção de mulheres idosas que moram sozinhas inscritas no PSF de Maceió mostra-se maior que a obtida pela Pesquisa Nacional por Amostra de Domicílios (PNAD) para o Nordeste $(10,8 \%) .{ }^{22} \mathrm{~A}$ frequência de entrevistadas que residem com os filhos é inferior ao relatado pelo PNAD (51,6\%). ${ }^{22}$ Este fato pode ocorrer por razões socioeconômicas ou características culturais. Embora a família, de certa forma, usufrua do rendimento do idoso, o suporte intergeracional funciona no sentido oposto. À medida que o idoso contribui com sua renda, termina promovendo a integração da família e, também, seu próprio bem-estar. ${ }^{23}$

Em geral, idosos que moram em domicílios multigeracionais tendem a ser mais pobres e ter maior grau de dependência, enquanto que idosos com melhor nível socioeconômico tendem a morar em domicílios unigeracionais ou sozinhos, têm menor grau de dependência e são mais preparados para não dependerem de outros. ${ }^{24} \mathrm{Ao}$ longo dos anos, o número de idosos que moram sozinhos vem crescendo sistematicamente. ${ }^{23}$ 
Em relação à escolaridade, verifica-se alto percentual de entrevistadas não alfabetizadas, seguido daquelas com um a dois anos de estudo. A PNAD, ${ }^{22}$ para Alagoas, revela taxa de analfabetismo e de apenas um ano de instrução na ordem de $60,6 \%$, valor próximo ao obtido nas idosas matriculadas no PSF de Maceió. A média nacional de idosos com idades de 60 anos ou mais, sem instrução e menos de um ano de estudo é de $33,5 \% .{ }^{22}$ A média de anos de estudo em idosos no Brasil é de 3,8 anos. Os anos de escolaridade no Brasil são uma boa aproximação das condições socioeconômicas e uma informação relativamente fácil de ser obtida, além do fato que a maior escolaridade se relaciona com a melhor qualidade de vida e longevidade. Romero ${ }^{25}$ declara que a chance de um idoso não alfabetizado autoavaliar de forma negativa a sua saúde é $60 \%$ maior em relação aos idosos alfabetizados. Além disso, quanto menor a renda per capita, maior a taxa de analfabetismo entre idosos. $\mathrm{O}$ rendimento percapita neste estudo foi maior para idosas com menos de um salário mínimo, enquanto que a PNAD relata o valor de $33,0 \%$ para o Nordeste, condição esta associada à situação de pobreza. ${ }^{22}$

A prevalência de entrevistadas que fumam no presente estudo é inferior a de ex-fumantes. No Brasil, o Sistema de Cadastramento e Acompanhamento de Hipertensos e Diabéticos (HiperDia), criado em 2002 pelo Ministério da Saúde, tinha $9.917(73,07 \%)$ pessoas do sexo feminino cadastradas no sistema, em Maceió, no mês de dezembro de 2009; destas, 36,4\% encontram-se na faixa etária de 60-79 anos. ${ }^{26} \mathrm{O}$ tabagismo em Maceió, segundo o registro no sistema HiperDia para esta faixa etária, foi de $17,5 \%$.

Como em outros estudos em idosos, a hipertensão arterial foi a comorbidade mais prevalente. A segunda morbidade autorreferida mais citada foi artrite/reumatismo/artrose. Embora a prevalência dessa doença entre idosas (60 + anos de idade) na PNAD/2003 tenha sido de $33,0 \%$ (um problema de saúde pública), não recebe a devida atenção, sendo tratada como doença individual. ${ }^{27,23}$ Sabe-se que é uma das principais responsáveis pela limitação de atividades no idoso. ${ }^{28}$ Os achados deste estudo são corroborados por Machado et al. ${ }^{29}$, que apontam a artrite como a segunda morbidade após a hipertensão arterial. Os autores, em estudo de base populacional do Projeto Bambuí, avaliando 1.606 pessoas, relataram prevalência de reumatismo diagnosticada por médicos de $25,3 \%$, e de sintomas clínicos nas mãos e joelhos em $44,2 \%$. Ambas as prevalências são mais frequentes no gênero feminino, na razão de 3:1. Na maioria das cidades da Pesquisa SABE, ao menos uma em cada três idosas informaram ter artrite e a prevalência para o sexo feminino foi de $39,6 \%{ }^{21}$

A prevalência e a incidência de $\mathrm{DM}$ vêm aumentando na população devido ao crescimento do extrato idoso, da urbanização e industrialização, além do aumento da obesidade, da inatividade física e do crescimento de sobrevida dos diabéticos. ${ }^{30} \mathrm{~A}$ frequência de $\mathrm{DM}$ autorreferido foi superior ao percentual de mulheres, na mesma faixa etária do estudo, cadastradas no Sistema HiperDia, em Maceió (29,6\%).

$\mathrm{Na}$ avaliação de sobrepeso/obesidade, o presente estudo encontrou percentuais em idosas na faixa de 60-69 anos um pouco menor que nos grupos cadastrados no Sistema HiperDia/Maceió $(43,1 \%)$, o que representa um desafio à saúde pública local. O excesso de peso é um dos principais fatores de risco de uma série de doenças crônicas nãotransmissíveis. Abrantes et al. ${ }^{31}$ corroboram os achados de Maceió: utilizando dados da Pesquisa sobre Padrões de vida (PPV) nas regiões Nordeste e Sudeste, encontraram declínio de obesidade em idosas a partir de 70 anos.

Outro dado preocupante é a prevalência de baixo-peso na faixa etária 70-79 anos. Essa diminuição pode ser atribuída ao declínio da massa muscular corporal, massa óssea e da quantidade de gordura corporal, que tende a diminuir após 70 anos. ${ }^{32} \mathrm{~A}$ CC é uma importante medida de avaliação de gordura abdominal. Neste estudo, a maior parte das idosas apresentaram CC $\geq 80 \mathrm{~cm}$ e quase a metade na faixa etária de 60 69 anos encontrava-se com CC $\geq 88 \mathrm{~cm}$, o que pode indicar risco para doença cardiovascular elevado ou muito elevado, respectivamente (WHO, 2000). $\cdot{ }^{14}$ Contudo, esses resultados devem 
ser interpretados com cautela, pois dados pertinentes a indivíduos idosos são escassos e não está claro se eles apresentam os mesmos riscos que indivíduos mais jovens. ${ }^{21}$

Há suspeita de depressão leve a moderada. Bós \& Bós, ${ }^{33}$ utilizando microdados da PNAD/1998, ao perguntarem aos idosos "têm depressão?", obtiveram $12,5 \%$ de resposta afirmativa, sendo maior nas mulheres e na faixa etária menor para maior (60-69 e 70-79 anos), 16,3\% e 15,5\%, respectivamente. Gazalle et al., ${ }^{34}$ em estudo de base populacional, relatam que os sintomas depressivos são maiores nas mulheres, nos idosos, naqueles com baixa escolaridade, sem trabalho remunerado, fumantes ativos e que sofreram perdas de familiares ou pessoas próximas.

No presente estudo, a frequência de entrevistadas que se apresentaram com dependência funcional moderada para a realização das AIVD foi inferior aos dados obtidos na frequência de incapacidadefuncional moderada em mulheres idosas na PNAD/2003, em Maceió (37,3\%). ${ }^{27}$ Ramosafirmaqueoconceitode saúde válido para o idoso está atrelado à autonomia. ${ }^{6} \mathrm{~A}$ baixa prevalência de dependência parcial pode ser decorrente da metodologia aplicada na seleção, excluindo aquelas que apresentaram incapacidade física.

Avaliadas pelo IPAQ, cerca de metade das entrevistadas foi considerada ativa ou muito ativa e outra metade, insuficientemente ativa ou sedentária. A porcentagem de sedentarismo encontrado foi inferior ao obtido na população feminina do HiperDia, Maceió (43,5\%). Neste estudo houve maior percentual de idosas ativas devido aos critérios de inclusão das participantes. Segundo Matsudo et al., ${ }^{18}$ a inatividade física (sedentarismo) é o fator de risco de doenças crônicas não-transmissíveis mais prevalente na população.
Muitos dos efeitos deletérios do envelhecimento são secundários à falta de atividade física regular, que acabam afetando negativamente a saúde, a capacidade funcional e a qualidade de vida. ${ }^{35}$ As evidências disponíveis sugerem associação inversa entre atividade física e morbimortalidade por doenças crônicas nãotransmissíveis, sobretudo hipertensão, artrose e diabetes.

\section{CONCLUSÕES}

Os dados encontrados para as idosas matriculadas no PSF de Maceió apontam que se trata de pessoas com baixa escolaridade, baixa renda e com percepção de saúde regular/ruim. Há elevado número de morbidades autorreferidas, sendo a hipertensão arterial, artrite/reumatismo/artrose e diabetes mellitus as mais frequentes. Metade delas se encontra em risco nutricional para doenças (obesidade ou baixopeso). Parte está com suspeita de depressão leve/ moderada (41,0\%), 50,7\% são sedentárias ou insuficientemente ativas e $12,7 \%$ apresentam dependência funcional para as atividades instrumentais da vida diária.

Estes dados visam a contribuir para a formulação de políticas públicas pelos gestores, de acordo com a realidade identificada no estudo. Os programas devem ser capazes de prevenir ou atenuar a incidência de DCNT, que com muita frequência geram incapacidade, reduzir as incapacidades presentes e combater o sedentarismo. Em simultâneo, podem promover ações para garantir que aqueles que vivam mais de 60 anos possam fazê-lo com dignidade e qualidade de vida. 


\section{REFERÊNCIAS}

1. Carvalho JAM, Garcia RA. O envelhecimento da população brasileira: um enfoque demográfico. Cad Saúd Públic 2003; 19(3): 725-33.

2. Instituto Brasileiro de Geografia e Estatística( IBGE). Projeção da população do Brasil por sexo e idade (1980-2050). Rio de Janeiro: IBGE; 2008. [ Acesso em 04 set 2009]. Disponível em URL: < http://www.ibge.gov.br>

3. Fundação Instituto Brasileiro de Geografia e Estatística. Indicadores sociodemográficos e de saúde no Brasil. Rio de janeiro : IBGE; 2009. [ Acesso em 04 set 2009] Rio de Janeiro: IBGE. Disponível em URL: < http:// www.ibge.gov.br $>$

4. Ministério da Saúde( Brasil). Agência Nacional de Saúde Suplementar (ANS). Taxa de cobertura de planos de saúde. [ Acesso em 03 set 2009].Disponível em URL: < http:// anstabnet.ans.gov.br/tabcgi.exe?dados/ TABNET_TX.def $>$

5. Veras RP, et al. Características demográficas dos idosos vinculados ao sistema suplementar de saúde no Brasil. Rev Saúd Pública 2008; 42(3): 497-502.

6. Ramos LR. Determinantes do envelhecimento saudável em idosos residentes em centro urbano: Projeto Epidoso. Cad Saúd Pública 2003; 19(3): 793-8.

7. Pinheiro RS, Travassos C. Estudo da desigualdade na utilização de serviços de saúde por idosos em três regiões da cidade do Rio de Janeiro. Cad Saúd Pública 1999; 15(3): 487-96.

8. Menezes TN, Souza JMP, Marucci MFN. Avaliação do estado nutricional dos idosos residentes em Fortaleza/CE: o uso de diferentes indicadores antropométricos. Rev Bras Cineantropometria \& Desempenho Humano 2008; 10(4): 315-22.

9. Spirduso WW. Dimensões físicas do envelhecimento. São Paulo: Manole; 2005. 482p.

10. Ministério da Saúde. Secretaria Executiva. Caderno de Informações de Saúde. [ Acesso em 03 set 2009].Disponível em URL: < http:// tabnet.datasus.gov.br/tabdata/cadernos/ al.htm >

11. Gordon CC, Chumlea WC, Roche AF. Stature, recumbent length, and weight. In: Lohman TG, Roche AF, Martorell R. Anthropometric standardization reference manual. Illinois: Human Kinetics Books; 1988.
12. Organização Pan-Americana da saúde (OPAS). SABE : Saúde, Bem-estar e Envelhecimento. [ Acesso em 23 jul 2007]. Disponível em URL: < http://www.opas.org.br/ publicmo.cfm? codigo $=70>$.

13. Callaway WC, et al. Circumferences. In: Lohman TG, Roche AF, Martorell R.

Anthropometric standardization reference manual. Illinois: Human Kinetics Books; 1988.

14. World health organization (WHO). Report of a Consultation. Obesity: prevening and managing the global epidemic. Geneva:Technical Report Series 894; 2000.

15. Yesavage JA, et al. Development and validation of geriatric depression screening scale: a preliminary report. J Psychiatr Res 1983; 17(1): 37-49.

16. Stoppe Júnior A, Jacob Filho W, Louza Neto MR. Avaliação de depressão em idosos através da Escala de Depressão Geriátrica: resultados preliminaries. Revi APB-APAL 1994 out-dez; 16(4): 149-53.

17. Lawton MP, Brody EM. Assessment of older people: self-maintaining and instrumental activities of daily living. Gerontologist 1969; 9(3): 179-186.

18. Matsudo SM, et al. Questionário Internacional de Atividade Física (IPAQ): estudo de validade e reprodutibilidade no Brasil. Rev Bras Ativ Fis Saúde 2001; 6(2): 5-18.

19. Centro de Estudos do Laboratório de Aptidão Física de São Caetano do Sul (CELAFISCS). Projeto Longitudinal do envelhecimento e aptidão Física de São Caetano do Sul. Disponível em URL: < http:// www.celafiscs.institucional.ws em 01 de agosto de 2009.

20. Center for Disease Control and Prevention (CDC). Promoting physical activity: a best buy in public. 2000.[ Acesso 04 set 2009]. Disponível em URL: http://www.cdc.gov

21. SABE. Saúde, Bem-Estar e Envelhecimento. O projeto sabe no município de São Paulo: uma abordagem inicial. Brasília: Organização PanAmericana da Saúde; 2003. 255p.

22. Instituto Brasileiro de Geografia e Estatística. Pesquisa Nacional por Amostra de Domicílios (PNDA, 2006-7). Rio de Janeiro: IBGE; 2007. Disponível em URL: < http:// www.ibge.gov.br $>$. 
23. Rede Interagencial de Informações para Saúde. Informe de situação e tendências: demografia e saúde/Rede Interagencial de Informações para Saúde. Brasília: Organização Pan-Americana de Saúde; 2009.

24. Coelho Filho JM, Ramos LR. Epidemiologia do envelhecimento no Nordeste do Brasil: resultados de inquérito domiciliar. Rev Saúd Pública 1999; 33(5): 445-53.

25. Romero DE. Diferenciais de gênero no impacto do arranjo familiar no status de saúde dos idosos brasileiros. Ciênc e Saúde colet 2002; 7(4):777-794.

26. DATASUS. Relatórios 2009. [ Acesso em set 2009].Disponível em URL: http:// www.hiperdia.datasus.gov.br.

27. Instituto Brasileiro de Geografia e Estatística(IBGE). Pesquisa Nacional por Amostra de Domicílios . Rio de Janeiro: Fundação IBGE; 2003. [Acesso em $1 \mathrm{dez}$ 2009].Disponível em URL: < http:// www.ibge.gov.br >

28. Lebrão ML, Laurenti R. Saúde, bem-estar e envelhecimento: o estudo SABE no Município de São Paulo. Rev Bras Epidemiol 2005; 8(2): 127-41.

29. Machado GPM, Barreto SM, Passos VMA, Lima-Costa MFF. Projeto Bambuí: prevalência de sintomas articulares crônicos em idosos. Rev Associação Méd Bras 2004; 50(4): 367-72.

30. Freitas EV. Diabetes mellitus. In: Freitas EV, Cançado FAX, Doll J, Gorzoni ML. Tratado de Geriatria e Gerontologia. 2 ed. Rio de Janeiro: Guanabara Koogan; 2006. p. 776-786.

31. Abrantes MM, Lamounier JA, Colosimo EA. Prevalência de sobrepeso e obesidade nas regiões nordeste e sudeste do Brasil. Rev Associação Méd Bras 2003; 49 (2): 162-6.

32. Noppa H, et al . Longitudinal studies of anthropometric data and body composition. The American Journal of Clinical Nutrition 1980; 33: 155-62.

33. Bós AMG, Bós AJG. Fatores determinantes e consequências econômicas da depressão entre os idosos no Brasil. Rev Bras Ciênc Envelhecimento Humano 2005; 2(2): 36-46.

34. Gazelle FK, Lima MS, Tavares BF, Hallal PC. Sintomas depressivos e fatores associados em população idosa no sul do Brasil. Rev Saúd Pública 2004; 38(3): 365-71.

35. Matsudo SM. Atividade física na promoção da saúde e qualidade de vida no envelhecimento. Rev. Bras. Educ. Fís. Esp 2006; Supl.5: S135-37. 